\title{
A model of knowledge management system and early warning system (KMS@EWS) for clinical diagnostic environment
}

\begin{abstract}
Early warning system (EWS) is a technology to mitigate risk in multi disciplinary areas. Issues on timeliness for timely reporting are still regarded as a main challenge in EWS. Therefore in this paper, we suggest the model of the integration between knowledge management system (KMS) and EWS known as KMS@EWS for clinical diagnostics (CD) environment. The integration model is to combine the advantage of KM system with the EWS functionalities and its components. The proposed model is based on empirical study by using literatures on KMS and EWS. We synthesize the findings of KMS and EWS model of integration. To demonstrate the application of this model, we propose into CD environment as a platform KMS@EWS system implementation. Our propose model can provide early warning when any abnormalities or peculiar pattern of disease and symptoms arise and detected during the interaction between physicians and patients. Thus, this model can support the managing of data and information for timely reporting and detection by providing decision facilitation thru early warning during the $\mathrm{CD}$ processes.
\end{abstract}

Keyword: Knowledge management; Knowledge management system; Early warning; Early warning system; Clinical diagnostic environment 